IOS Press

\title{
Mechanism of Bushen Jianpi decoction in preventing and treating osteoporosis caused by aromatase inhibitors in breast cancer treatment
}

\author{
Xu-Hui Huang ${ }^{\mathrm{a}}$, Rong-Hua Liang ${ }^{\mathrm{b}}, \mathrm{Le} \mathrm{Su}^{\mathrm{b}}$, Wei Guo ${ }^{\mathrm{b}}$ and Chang-Jun Wang ${ }^{\mathrm{a}, *}$ \\ ${ }^{a}$ Department of Traditional Chinese Medicine, Guangdong General Hospital, Guangdong Academy of Medical \\ Sciences, Guangzhou 510080, Guangdong, China \\ ${ }^{\mathrm{b}}$ Guangzhou University of Chinese Medicine, Guangzhou 510403, Guangdong, China
}

\begin{abstract}
.
PURPOSE: Research on the mechanism of Bushen Jianpi decoction (BJD) for preventing and treating osteoporosis caused by aromatase inhibitors (AI) during treatment for breast cancer resection.

METHODS: An ovariectomized mouse model was established using random division into 6 groups: a sham ovariectomized group, a blank control group, a control group, an alendronate group, a BJD group, and a drug combination group. Mice breast cancer cell lines (4T1) were cultured and seeded into the armpits of 6 groups of BALB/c mice. The mouse breast cancer postoperative model was built when resecting the tumor after 3 weeks following seeding tumor. After 1 weeks, the 6 groups of mice were given different drugs. Then the following analyses were made: estradiol (E2) levels and alkaline phosphatase (ALP) levels in the serum; detection of in vitro bone density and calcium and bone phosphorus content; tumor pathology and immunohistochemistry detection.

RESULTS: The results suggested that BJD decreased levels of ALP in ovariectomized mice, and there was a trend for improved bone loss. BJD strengthened the trend of alendronate to improve bone loss, improved bone density, bone calcium and phosphorous, and reduced ALP. BJD had a certain role on the promotion of the expression of estrogen receptors (ERs) in the relapse of the tumor tissue.

CONCLUSIONS: Combined therapy with BJD and alendronate can act synergistically against osteoporosis, which was possibly related to a reduced bone conversion rate through inhibiting bone resorption. BJD may block the MAPK signal pathway in breast cancer cells, increasing the expression of ERs and making cancer cells sensitive to endocrine treatment.
\end{abstract}

Keywords: Bushen Jianpi decoction, breast cancer, AI treatment, osteoporosis

\section{Introduction}

Endocrine therapy can reduce the secretion of estrogen in the body, reducing its levels or blocking its pathway, thereby inhibiting the growth and proliferation of

${ }^{*}$ Corresponding author: Chang-Jun Wang, Department of Traditional Chinese Medicine, Guangdong General Hospital, Guangdong Academy of Medical Sciences, No. 106 of Zhongsaner Road, Yuexiu District, Guangzhou 510080, Guangdong, China. Tel.: +86 208382 7812; Fax: +86 208382 7812; E-mail: gzwchj@163.com. tumor cells. This is an important means of comprehensive treatment for breast cancer [1]. Since aromatase inhibitors (AIs) are the first choice of drug for postmenopausal patients under endocrine therapy, patients using AIs often suffer from fractures, muscle and joint pain, and other symptoms that seriously affect quality of life. There is an urgent need to address and prevent this problem [2,3]. The key to bone-related events is that bone metabolic imbalances are caused by the decrease in estrogen levels [4]. Therefore, it is difficult to treat endocrine therapy in breast cancer-related os-

ISSN 1574-0153/17/\$35.00 (C) 2017 - IOS Press and the authors. All rights reserved

This article is published online with Open Access and distributed under the terms of the Creative Commons Attribution Non-Commercial License (CC BY-NC 4.0). 
teoporosis. Breast cancer should be considered when treating osteoporosis, which is different from ordinary osteoporosis treatment.

There is the unique advantage through the whole concept and dialectical theory of the governance of traditional Chinese medicine towards such disorders, in which patients can have better treatment compliance. After breast cancer surgery, patients usually lack blood; thus, it is difficult for blood to nourish meridians. Due to endocrine therapy after surgery, lack of appetite, kidney essence deficiency, yin and yang disharmony, menstruation failure, as well as the inability of bones to produce marrow, occurs; which makes the bones weak and susceptible to fracture. Due to this, many breast cancer patients who use endocrine therapy have many visible spleen and kidney deficiency problems. Therefore, tonifying the kidneys and spleen is an important means of improving endocrine treatmentrelated osteoporosis.

This study created a complete model of breast cancer and resection using ovariectomized mice. AIs were given orally to simulate this clinical situation, leading to endocrine therapy for breast cancer osteoporosis after resection. We used Bushen Jianpi decoction (BJD) for treating osteoporosis caused by AIs to provide experimental evidence for the clinical application of BJD.

\section{Materials and methods}

\subsection{Materials}

RPMI-1640 medium, fetal bovine serum and $0.25 \%$ trypsin were obtained from Gibco (Shanghai, China); penicillin G, streptomycin and BSA were obtained from Sigma (Shanghai, China); serum E2 and ALP kits were obtained from Cloud-Clone company; alendronate sodium and anastrozole standards were provided by the Guangdong Provincial Institute for Drug Control (Guangzhou, China); rabbit anti-human estrogen receptor (ER) and progesterone receptor (PR) antibodies were obtained from the Three Pagodas Company (Guangzhou, China); SABC immunohistochemical kits and hematoxylin and eosin (H\&E) staining kits were obtained from the Boster Company (China). 4T1 murine breast cancer cells were supplied by the College of Pharmacy of Sun Yat-Sen University (China).

\subsection{BJD preparation}

The BJD preparation consisted of $20 \mathrm{~g}$ of xianmao (Rhizoma curculinis), $20 \mathrm{~g}$ of Epimedium, $15 \mathrm{~g}$ of baji- tian (Radix morindae officinalis), $20 \mathrm{~g}$ of yunling (Poria), $20 \mathrm{~g}$ of baishu (Rhizoma atractylodis macrocephalae), $20 \mathrm{~g}$ of huaishan (Rhizoma dioscoreae), $15 \mathrm{~g}$ of gusuibu (Rhizoma drynariae), forging longgu (Os Draconis), $20 \mathrm{~g}$ of muli (Concha ostreae), $15 \mathrm{~g}$ of poguzhi (Fructus psoraleae), $10 \mathrm{~g}$ of dilong (Pheretima), $6 \mathrm{~g}$ of tubiecong (Eupolyphaga seu steleophaga), and other components. The equivalent dose of BJD in mice was $33.5 \mathrm{~g} / \mathrm{kg}$, according to the "Experimental Pharmacology methodology".

\subsection{Preparation of anastrozole solution}

One milligram of anastrozole was added into $100 \mathrm{ml}$ of distilled water, ultrasonically vibrated to form a suspension, kept in the dark at $4^{\circ} \mathrm{C}$, and shaken well before use.

\subsection{Preparation of alendronate sodium solution}

Ten milligrams of alendronate sodium was added into $200 \mathrm{ml}$ of distilled water, ultrasonically vibrated to form a suspension, kept in the dark at $4^{\circ} \mathrm{C}$, and shaken well before use.

\subsection{Cell culture}

4T1 breast cancer cells were cultivated at $37^{\circ} \mathrm{C}$ in an incubator with 5\% CO2. Cell logarithmic growth phase, trypsin, and 1:3 passage.

\subsection{Animal treatments}

Six-week-old SPF female BALB/c mice were purchased from the Experimental Animal Hayes Lake LLC (Shanghai, China; a certificate of 2,013,001,801, 067). During the experiment, they were raised at the SPF Animal Laboratory of the Experimental Animal Center in the First Hospital Affiliated to Sun Yat-Sen University, Guangzhou, China (qualification certificate: SYXK [Guangdong] 2010-0108). Ninety female mice were randomly divided into six equal groups: sham ovariectomized group, blank control group, control group, alendronate group, BJD group and drug combination group. In addition, all females in the sham ovariectomized group underwent ovariectomy surgery and were ovariectomized. Eight weeks after ovariectomy, the six groups were inoculated with tumor cells to create the model. 4T1 cells were seeded into the right armpits of all mice. Three weeks after inoculation, mice with an epidermal tumor diameter $>0.3 \mathrm{~cm}$ 
were selected and anesthetized through pentobarbital intraperitoneal injection. Subsequently, under sterile conditions, all visible tumor tissues were resected and a suture was used to stop the bleeding. At one-week post-operative, the incisions were dry, the mice appeared healthy and no tumor recurred. This situation is considered successful after breast cancer surgery. Ten mice in each group were drenched with appropriate drugs: the sham ovariectomized group and control group were given a $20 \mathrm{ml} / \mathrm{kg}$ dose of BW/d normal saline; the control group underwent $0.15 \mathrm{mg} / \mathrm{kg}$ $\mathrm{BW} / \mathrm{d}$ gavage anastrozole suspension, the alendronate group were fed with $0.15 \mathrm{mg} / \mathrm{kg} \mathrm{BW} / \mathrm{d}$ of anastrozole and $1.5 \mathrm{mg} / \mathrm{kg} \mathrm{BW} / \mathrm{d}$ of alendronate sodium suspension; the BJD group were fed with $0.15 \mathrm{mg} / \mathrm{kg} \mathrm{BW} / \mathrm{d}$ of anastrozole suspension and $33.5 \mathrm{~g} / \mathrm{kg} \mathrm{BW} / \mathrm{d}$ of BJD; the drug combination group were fed with $0.15 \mathrm{mg} / \mathrm{kg}$ $\mathrm{BW} / \mathrm{d}$ of anastrozole suspension, $1.5 \mathrm{mg} / \mathrm{kg} \mathrm{BW} / \mathrm{d}$ of alendronate suspension and $33.5 \mathrm{~g} / \mathrm{kg} \mathrm{BW} / \mathrm{d}$ of BJD. All mice were treated for four consecutive weeks.

\subsection{Specimen collection}

After the last administration at 24 hours, orbital blood was taken from mice. Serum was centrifuged (3,000 rpm, five minutes) and stored in a refrigerator at $-80^{\circ} \mathrm{C}$, which would be used for the subsequent detection of estradiol (E2) and ALP. After collecting blood, mice were euthanized by cervical dislocation. Under sterile conditions, tissue was taken from the site of tumor tissue inoculation, and was weighed and soaked overnight in $4 \%$ paraformaldehyde for pathological analysis. Bilateral femurs and tibias were removed from the mice. The left femur was kept for in vitro bone density detection, while the right femur and tibia bones were used for calcium and phosphorus determination.

\subsection{Hormone level detection}

Analysis of E2 levels in serum was performed using an automated chemiluminescence immunoassay analyzer.

\subsection{Assessment of osteoporosis index and in vitro bone density testing}

The above tests were performed using a GE Lunar Prodigy dual-energy X-ray absorptiometry (USA), with its accompanying small animal bone density test software, respectively. The bone mineral density
Table 1

Comparison of groups' E2 (mean \pm standard deviation)

\begin{tabular}{lcl}
\hline Group & $\mathrm{n}$ (number) & E2 $(\mathrm{pg} / \mathrm{ml})$ \\
\hline Sham ovariectomy group & 10 & $58.98 \pm 7.89$ \\
Blank control group & 10 & $25.43 \pm 5.36^{\mathrm{a}}$ \\
Control group & 10 & $12.83 \pm 3.05^{\mathrm{ab}}$ \\
Alendronate group & 10 & $11.52 \pm 1.99^{\mathrm{a}}$ \\
BSJPD group & 10 & $12.93 \pm 2.65^{\mathrm{ab}}$ \\
Combined group & 10 & $13.35 \pm 3.06^{\mathrm{ab}}$ \\
\hline
\end{tabular}

a: represents compared to sham castrated group, $P<0.05$; b: represents compared with the blank control group, $P<0.05$; c: represents compared with the control group, $P<0.05$. d: represents compared with the alendronate group, $P<0.05$. e: represents compared to the kidney and spleen prescription group, $P<0.05$.

(BMD) of each left femur bone was analyzed. Serum alkaline phosphatase detection was performed with an automatic biochemical analyzer using the AMP-buffer method recommended by the International Federation of Clinical Chemistry (IFCC). Calcium and bone phosphorus content were determined by ICP emission spectrometry.

\subsection{Tumor pathology and immunohistochemistry detection}

Tissue dehydration; tissue embedding; paraffin tissue slices, spread sheet, fishing slices, toasted slices; $\mathrm{H} \& \mathrm{E}$ staining; immunohistochemical staining. Immunohistochemical results on microscopic sections were judged according to nuclei appearing yellow or brown and representing positive cells. Five horizons were randomly selected, and the total number of tumor cells and positive cells were counted. Positive cells present at $<5 \%$ were judged as negative (-), a positive rate of $<10 \%$ were judged as weak positive $(+), 10$ $50 \%$ were judged as positive $(++)$, and $>50 \%$ were judged as strong positive (+++).

\subsection{Statistical analysis}

Measurement results are expressed as mean \pm standard deviation. SPSS 19 software package was used for statistical analysis, and measurement data differences within each group were compared using $t$-test. Analysis of variance was used to compare measurement data between groups. Count data between groups were compared using Chi-square tests. A $P$-value $<$ 0.05 was considered statistically significant. 


\section{Results}

\subsection{Test results of hormone levels}

E2 levels in each drug group were lower in the sham ovariectomized group and blank control group, and there was a significant difference $(P<0.05)$ between each group. Results are shown in Table 1. Results indicate that the ovariectomy model was successful, and that the mice created a good simulation of human menopausal status after ovariectomy. Endocrine therapy can further reduce E2 levels. E2 levels between each treatment group were not significantly different $(P>0.05)$. Table 1 illustrates that each regimen did not have a significant impact on E2 levels after ovariectomy.

\subsection{Results of assessment indicators of osteoporosis}

There was no significant difference $(P>0.05)$ in BMD, ALP content, and bone calcium and phosphorus content between the control group and blank group; but this situation is worse than the sham ovariectomy group, and there was a significant difference $(P<$ $0.05)$. Although there was no significant difference in each bone indices in the control group and blank control group, indicators in the control group were slightly worse than in the blank control group (Table 2). Results suggest that bone mineral density in ovariectomized mice in each group, osteocalcin, and bone phosphorus levels were reduced, while ALP increased; thus, the ovariectomy-induced osteoporosis model was successful and the administration of anastrozole intensified this trend in osteoporosis.

Compared with the sham ovariectomy group, each use of alendronate regimen (alendronate group and drug combination group) revealed no significant difference $(P>0.05)$ for BMD and osteocalcin. However, with non-alendronate regimen groups (blank control group and BJD group), there was a significant difference $(P<0.05$, Table 2$)$. Results revealed that alendronate can increase bone density and calcium, as well as phosphorus bone content, and reduce the role of ALP in ovariectomized mice.

In contrast, for BMD, ALP content, bone calcium and phosphorus content, the BJD group and sham ovariectomized group were significantly different $(P<0.05)$. However, the blank control group revealed no significant difference $(P>0.05)$ in BMD, bone calcium and phosphorus (Table 2). Results suggest that although BJD did not significantly improve bone density or bone calcium and phosphorus in the short term, bone loss was not significantly reduced; and the BJD group did show minor improvements in bone density and bone calcium and phosphorous. This suggests that BJD can decrease ALP in ovariectomized mice, which can lead to a trend of improved bone loss. When comparing with bone density, calcium, bone $\mathrm{P}$ and Serum ALP reacts to bone metabolism sensitivity beforehand.

For ALP content, there was no significant difference in bone mineral density and bone calcium and phosphorus content between the drug combination group and alendronate group $(P>0.05)$. However, the index of the drug combination group revealed a slight improvement (Table 2). These results suggest that BJD strengthened the trend of alendronate in improving bone loss; and that BJD and alendronate combined improved bone density, bone calcium and phosphorous, and reduced ALP, which achieved better levels than without ovariectomy.

\subsection{Tumor pathological and immunohistochemical results}

Before treatment, tumor tissues removed from each group was H\&E stained to reveal pleomorphic cells. There was a high proportion of nuclear transfer in more deeply stained nuclei, but there was no difference between groups (Fig. 1). These results indicate that the breast cancer model had has been successfully established.

Before treatment, ER immunohistochemical results in each group were shown to be negative. However, after treatment, recurrent tumors were resected from mice. Results of the non-use BJD group were negative, while the results part of using the BJD group was weakly positive (Table 3, Fig. 2). These results suggest that BJD has a certain role in promoting the expression of ERs.

Before treatment, PR immunohistochemical results in each group were shown to be negative. After treatment, in tumor tissues of relapsed excised mice, PR immunohistochemical results remained negative (Table 3 and Fig. 3). These results indicate that BJD had no impact on PRs.

\section{Discussion}

Bushen Jianpi Fang (a reinforcing kidney and strengthening spleen recipe) pays equal attention to 
Table 2

Comparison of bone metabolism and bone mineral density index in each group (mean \pm standard deviation)

\begin{tabular}{llllll}
\hline Groups & $\mathrm{n}$ & BMD $\left(\mathrm{mg} / \mathrm{cm}^{2}\right)$ & ALP $(\mathrm{ng} / \mathrm{ml})$ & Calcium $(\mathrm{mg} / \mathrm{g})$ & Bone $\mathrm{phosphorus}(\mathrm{mg} / \mathrm{g})$ \\
\hline Sham castrated group & 10 & $0.239 \pm 0.038$ & $4.86 \pm 1.01$ & $61.6 \pm 6.0$ & $30.46 \pm 3.45$ \\
Blank control group & 10 & $0.203 \pm 0.018^{\mathrm{a}}$ & $6.23 \pm 0.67^{\mathrm{a}}$ & $52.51 \pm 5.62^{\mathrm{a}}$ & $25.31 \pm 3.13^{\mathrm{a}}$ \\
Control group & 10 & $0.201 \pm 0.010^{\mathrm{a}}$ & $6.57 \pm 0.61^{\mathrm{a}}$ & $51.43 \pm 6.92^{\mathrm{a}}$ & $24.03 \pm 3.63^{\mathrm{a}}$ \\
Alendronate group & 10 & $0.234 \pm 0.010^{\mathrm{bc}}$ & $5.09 \pm 0.39^{\mathrm{bc}}$ & $58.87 \pm 6.00^{\mathrm{bc}}$ & $28.45 \pm 4.53^{\mathrm{c}}$ \\
BSJPD group & 10 & $0.207 \pm 0.009^{\mathrm{ad}}$ & $6.01 \pm 0.12^{\mathrm{acd}}$ & $52.19 \pm 5.14^{\mathrm{ad}}$ & $25.02 \pm 3.33^{\mathrm{a}}$ \\
The combined Groups & 10 & $0.237 \pm 0.007^{\mathrm{bce}}$ & $4.96 \pm 0.10^{\mathrm{bce}}$ & $59.23 \pm 3.83^{\mathrm{bce}}$ & $28.63 \pm 6.27^{\mathrm{bc}}$ \\
\hline
\end{tabular}

a: represents compared to sham castrated group, $P<0.05$; b: represents compared with the control group, $P<0.05$; c: compared with the control group, $P<0.05$. d: represents compared with the alendronate group, $P<0.05$. e: represents compared to the kidney and spleen prescription group, $P<0.05$.

Table 3

Estrogen receptor (ER) and progesterone receptor (PR) immunohistochemical expression in each group

\begin{tabular}{|c|c|c|c|c|c|}
\hline \multirow[t]{2}{*}{ Groups } & \multirow[b]{2}{*}{$\mathrm{n}$} & \multicolumn{2}{|c|}{ ER } & \multicolumn{2}{|c|}{ PR } \\
\hline & & Before treatment & After treatment & Before treatment & After treatment \\
\hline Sham castrated group & 10 & - & - & - & - \\
\hline Blank control group & 10 & - & - & - & - \\
\hline Control group & 10 & - & - & - & - \\
\hline Alendronate group & 10 & - & - & - & - \\
\hline BSJPD group & 10 & - & $-\sim+$ & - & - \\
\hline Combined group & 10 & - & $-\sim+$ & - & - \\
\hline
\end{tabular}

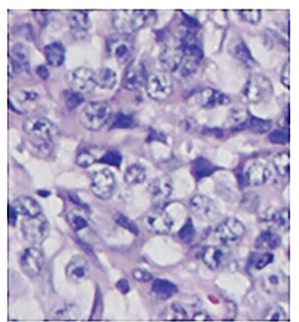

A: Sham castrated group

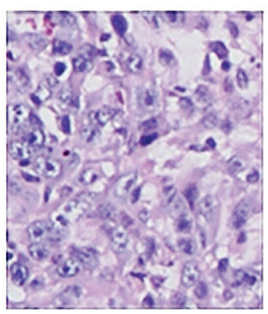

D: Alendronate group

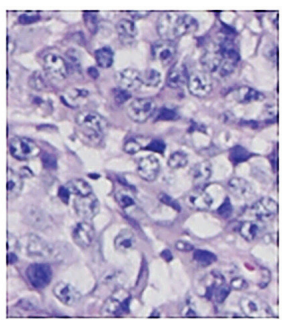

B: Blank control group

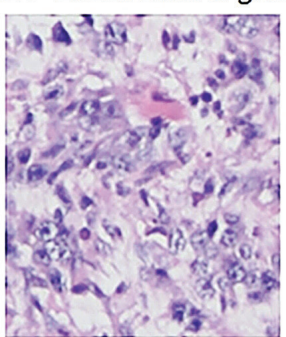

E : Kidney and spleen recipe group

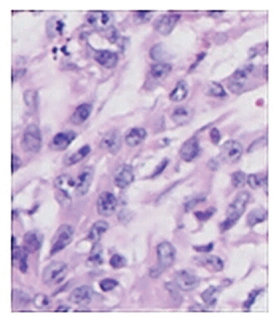

\section{C: Control group}

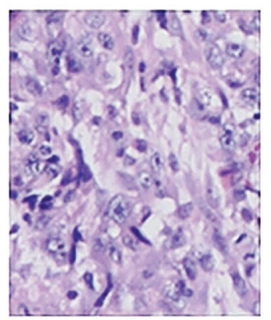

F: Combined group

Fig. 1. Before tumor treatment hematoxylin and eosin staining $(400 \times)$.

invigorating both the kidneys and spleen. Kidneyinvigorating herbal medicines, which reinforce the kidneys to strengthen bones, include Xianmao (Curculigo orchiodes), Xianlingpi (Herba epimedii), Duzhong (Cortex eucommiae), Bajitian (Morinda officinalis), etc. Spleen-invigorating herbal medicine, which replenish qi to invigorate the spleen, include Shan yao (Chinese Yam), Fuling (Poria cocos), Baizhu (large- head atractylodes rhizome), etc. Meanwhile, when combined with blood circulation (promoting and mass), resolving herbal medicines such as Dilong and Tubiechong can regulate qi-blood and improve local blood circulation. Combined with Duanlongmu and Duanmuli, it can nourish yin to suppress hyperactive yang, and astring to control and may even supply calcium directly; and combined with Huangbai, Zhimu, it 


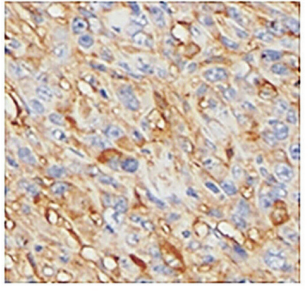

A: Sham castrated group

$$
(-)
$$

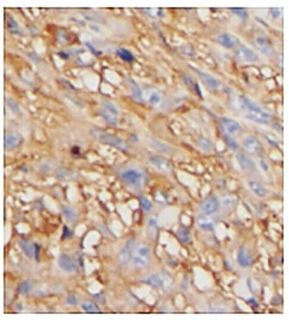

D: Alendronate group $(-)$

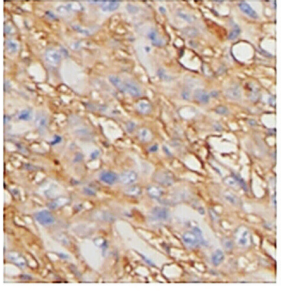

B: Blank control group

$$
(-)
$$

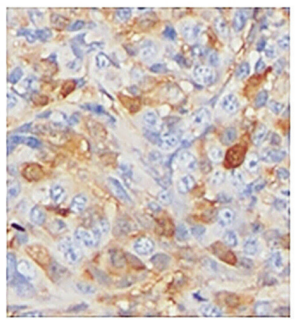

E: Kidney and spleen recipe group $(-\sim+)$

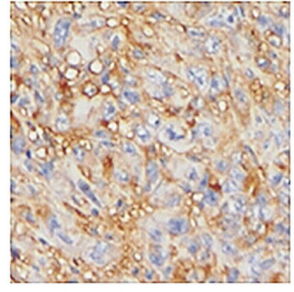

C: Control group (-)

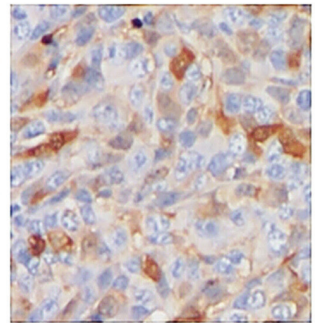

F: Combined group $(-\sim$

+ )

Fig. 2. Tumor estrogen receptors stained after treatment Immunohistochemical staining $(400 \times)$.

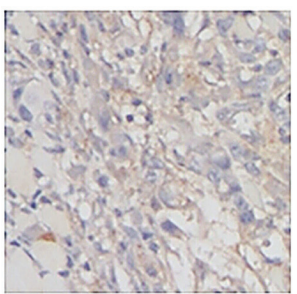

A: Sham castrated group $(-)$

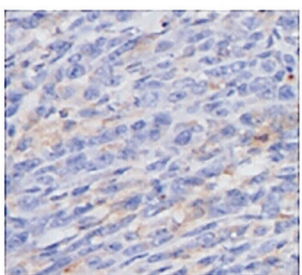

D: Alendronate group $(-)$

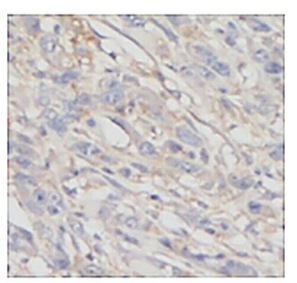

B : Blank control group $(-)$

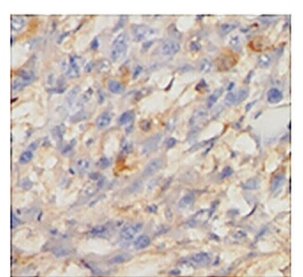

E : Kidney and spleen recipe group ( - )

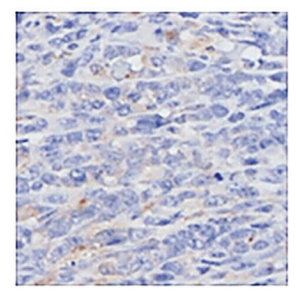

C: Control group (- )

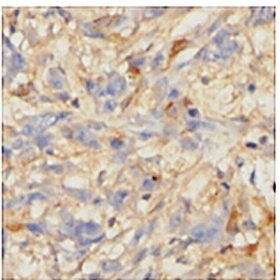

F : Combined group $(-)$

Fig. 3. Treatment tumor progesterone receptor immunohistochemical staining $(400 \times)$.

can clear away heat and nourish yin. Minor modifications are allowed according to the principles of treatment based on syndrome differentiation.

According to results, the level of ALP, bone mineral density, bone calcium and phosphorus in the sham ovariectomized group were all poorer than in the nonovariectomized groups. After ovariectomy, it is considered that estrogen secretion is reduced in mice; and this decrease in estrogen levels breaks hormonal imbalance, affecting the body's normal metabolism. The decrease in estrogen levels disrupts the dynamic equilibrium between bone formation and bone resorption; and the more the bone forms, the less the bone re-absorbs. As bone mineral density drops, the trabecular structure and bone mass formation increasingly declines, the balance of bone metabolism is broken, followed by 
a reduction in bone calcium, and eventually osteoporosis. This process involves an increase in the resorption of osteoclasts, but lower formation of osteoblasts. This results in a large number of osteoblast cells that are destroyed, breaking the homeostasis of bone remodeling $[5,6]$. Once this state is broken, ALP in damaged osteoblasts would be released into the blood or tissues, causing increased blood ALP activity, leading to a decrease in bone density, calcium and phosphorus [7].

Clinically, loss in the bone matrix due to endocrine therapy on post-operational breast cancer patients is usually treated with bisphosphonate. Thus, both in ovariectomy and AI treatment, the level of ALP in the groups that were administered with alendronate sodium was lower than the group that did not receive alendronate sodium, and the content of BMD and bone calcium significantly increased $(P<0.05$, Table 2$)$. These results show that alendronate sodium treatment has a therapeutic effect on bone loss caused by endocrine therapy in breast cancer [8], which is consistent with relevant literatures and researches.

BSJPD can significantly reduce the content of ALP and tend to prevent bone loss. This mechanism is considered to inhibit osteoclast activity and increase osteoblast activity. In this experiment, we transplanted breast cancer cells into ovariectomized mice. However, due to growth over time, there would have been tumor recurrence in some mice. As this tumor burden may have been great, it did not meet the ethical requirements for animal treatment. Thus, it may have been insufficient to consider medication after only four weeks of medication; at the same time, this does not rule out that tumors may have metastasized and caused bone destruction in the body. The BJD group and control group were not significantly different $(P>0.05)$ with regard to bone mineral density and bone calcium and phosphorus content, and BJD had no significant role in reducing bone loss. If it can increase postoperative medication time, bone mineral density, as well as bone calcium and phosphorus, may be observed to increase in the BJD group.

This study found that patients with bone loss can significantly decrease bone turnover markers after six months of treatment, although there was no significant change in bone density, owing to several months of lag. Thus, bone turnover markers would correspond to early changes, while bone mineral density would correspond to change in the latter. Therefore, serum ALP would usually be more sensitive in reflecting the level of bone activity than other indicators such as BMD, bone calcium and phosphorus. From the experiment, it was observed that the BJD group and control group were significantly different in ALP content $(P<0.05)$, suggesting that BJD can reduce bone turnover rate by inhibiting bone resorption, which tend to improve bone loss caused by endocrine treatment for breast cancer.

Although the drug combination group and alendronate group were not significantly different $(P>$ 0.05 ) in ALP content, BMD, and bone calcium and phosphorus, each index slightly improved. Compared with the alendronate group, ALP content in the drug combination group decreased, while BMD and bone calcium and phosphorus increased; and this contrast revealed a significant difference $(P>0.05$, Table 2$)$. Results indicate that drug combination therapy can increase BMD and bone mineral content, with the best efficacy in improving bone loss caused by endocrine treatment for breast cancer.

AI has now been developed to the third generation. Exemestane, letrozole and anastrozole are currently widely used in clinical medicine as typical representatives. Estrogen would promote the proliferation of breast cancer cells with ERs. At present, the ER analysis of breast cancer patients all revealed positive. Before menopause, estrogen mainly comes from the ovary. After menopause, $70 \%$ of the estrogen is dependent on androgens compounded by the adrenal and transformed from aromatase [9]. Meanwhile, the activity of aromatase in surrounding tissues is approximately $70 \%$ lower than in tumor tissues, and the proliferation of breast cancer cells is faster than in surrounding tissues $[10,11]$. In the human body, only 2 $5 \%$ of aromatase activity is not affected by AIs. Therefore, AIs can decrease the concentration of estrogen in the circulation and directly block tumor producing estrogen locally [12]. Accordingly, breast cancer patients who are hormone receptor-positive can obviously inhibit the recurrence and metastasis of tumors when given with AI therapy with confirmation of a postmenopausal state after surgery.

Studies have found that some E2/ER-dependent proliferation pathways of breast cancer can promote multiplication by regulating a series of oncogene-activated or anti-oncogene inactivated cells [13]. However, when ER-analysis produces negative results in breast cancer patients, this proliferation pathway would not rely on ERs. In such cases, tumor cells mainly rely on MAPK and PI3K/Akt pathways [14], and abnormal activation would promote cell proliferation. Thus, this kind of proliferation is not affected by estrogens, and patients with negative ER analysis would not benefit from taking AIs. The 4T1 cells in this experiment 
were ER-negative, showing insensitivity to anastrozole treatment; but the expression of ERs was upregulated after treatment with anastrozole and BJD. The reason may be is that BJD treatment caused ER expression in breast cancer cells to increase and the proliferation pathway to change from non-ER-dependent to ER-dependent. In this case, breast cancer cells would become sensitive to estrogen, and tumor proliferation may be inhibited under anastrozole therapy $[15,16]$.

In this experiment, the expression of ERs was upregulated after treatment with $\mathrm{BJD}$, and tumor cells became sensitive to estrogen. It was therefore inferred that BJD can inhibit the MAPK signal pathway in 4T1 cells and upregulate the expression of ERs. As the proliferation of tumor cells depends on the MAPK signal pathway, once estrogen is inhibited, tumor proliferation would also be inhibited. All medication groups in the experiment were PR-negative. Results revealed that when ERs were absent or weakly expressed, they could not regulate the expression of PRs; or alternately, the low level of ERs could not interact with the $\mathrm{PI} 3 \mathrm{~K} / \mathrm{Akt} / \mathrm{mTOR}$ signaling pathway, and therefore could not affect the expression of PRs.

In conclusion, this study suggests that BSJF is not an obvious anti-osteoporosis treatment over a short period of time, but it has a tendency to improve bone loss. Combined therapy with BJD, alendronate and anastrozol can act synergistically against osteoporosis. BJD does not affect E2 level in mice, and its auxiliary anti-tumor mechanism may block the MAPK signaling pathway in breast cancer cells; thus, increasing the expression of ERs and making cancer cells sensitive to endocrine treatment. Combined therapy to improve bone loss caused by endocrine treatment for breast cancer is not correlated with hormone levels, owing to the reduction in bone conversion rate through the inhibition of bone resorption. Finally, the means of improving the effect of endocrine therapy in breast cancer, preventing drug resistance and improving adverse reactions requires further studies.

\section{References}

[1] Zelnak AB, O'Regan RM. Optimizing endocrine therapy for breast cancer [J]. J Natl Compr Canc Netw. 2015; 13(8): e56e64.
[2] Chumsri S. Clinical utilities of aromatase inhibitors in breast cancer [J].Int J Womens Health. 2015; 7: 493-499.

[3] Van Asten K, Neven P, Lintermans A, et al. Aromatase inhibitors in the breast cancer clinic: focus on exemestane [J]. Endocr Relat Cancer. 2014; 21(1): R31-R49.

[4] Gobbi S, Rampa A, Belluti F, et al. Nonsteroidal aromatase inhibitors for the treatment of breast cancer: an update $[\mathrm{J}]$. Anticancer Agents Med Chem. 2014; 14(1): 54-65.

[5] Xiaodan Gai. The clinical observation of bone mineral density and total alkaline phosphatase in long-term supplement estrogen women with ovaries removed. Chin J Clin Rehabil. 2003; 7(5): 810 .

[6] Yu Du, Xiangling Tan. The study of the source of alkaline phosphatase in blood of rats with osteoporosis. Shandong Medi J. 2011; 51(19): 8-9.

[7] Ying Du, Jie Xie, Hewei Wei, et al. The clinical study of Bushen Jianpi Huoxue decoction in the treatment of osteoporosis. Chin J Clin Rehabil. 2005; 9(35): 108-110.

[8] Lin Ren, Xiaowei Qi. Sagopilone inhibits bone metastases and bone destruction in breast cancer by inhibiting tumor cell growth and bone absorption. Chin J Breast Dis. 2009; 3(5): 574-576.

[9] Szulc P, Delmas PD. Biochemical markers of bone turnover: potential use in the investigation and management of postmenopausal osteoporosis [J]. Osteoporos Int. 2008; 19(12): 1683-1704.

[10] Kurebayashi J. Current clinical trials of endocrine therapy for breast cancer [J]. Breast Cancer. 2007; 14(2): 200-214.

[11] Legault C, Maki PM, Resnick SM, et al. Effects of tamoxifen and raloxifene on memory and other cognitive abilities: cognition in the study of tamoxifen and raloxifene. J Clin Oncol. 2009; 27: 5144-5152.

[12] Kudachadkar R, O'Regan RM. Aromatase inhibitors as adjuvant therapy for postmenopausal patients with early stage breast cancer. CA Caneer J Clin. 2005; 55(3): 145-163.

[13] Xiaoning Tan, Zhi Zhou, Xiaolei Xie. The role of estrogen receptor signaling pathways in the development and treatment of breast cancer. Chin Bull Life Sci. 2011; 23(10): 969-974.

[14] Creighton CJ, Hilger AM, Murthy S, et al. Activation of mitogen-activated protein kinase in estrogen receptor alphapositive breast cancer cells in vitro induces an in vivo molecular phenotype of estrogen receptor alpha-negative human breast tumors. Cancer Res. 2006; 66(7): 3903-3911.

[15] Gee JM, Harper ME, Hutcheson IR, et al. The antiepidermal growth factor receptor agent gefitinib (ZD1839/Iressa) improves antihormone response and prevents development of resistance in breast cancer in vitro. Endocrinology. 2003; 144(11): 5105-5117.

[16] Bayliss J, Hilger A, Vishnu P, et al. Reversal of the estrogen receptor negative phenotype in breast cancer and rest oration of anti-estrogen response. Lincancer Res. 2007; 13(23): 70297036 